\title{
Editorial
}

\section{Impact Factor: No metric is perfect}

The Impact Factor has the hard task to measure the quality of a scientific journal and the impact on the community, based on the number of citations of articles from a given journal. Using this number, we may rank journals across different fields. However, this quality criterion rests on the assumption that its creator, Eugene Garfield, stated as: "The more frequently a journal's articles are cited, the more the world's scientific community implies that it finds the journal to be a carrier of useful information". This rank would actually help librarians to improve the management in their collections. However, since 1975, this number has become a fashionable and trendy topic that researchers pursue to escalate because of a wide range of reasons, including more chances for financial support. Thus, as expected, an index made for journal evaluation has switched to being a quantitative measure of scientific achievement and individual performance.

The evaluation of scientific quality is complex, as is the scientific community itself. Several other indexes have tried to accomplish a similar goal, but the Impact Factor has prevailed among others because Journals persist in reminding us about it. In consequence, where to publish is based mainly on the Journal's Impact Factor, influencing back on this number. Our journal has been recently granted with an Impact Factor of 0.206. This number represents the number of citations during the previous two years given the number of citable source items published during those years. The median for the Subject Area, Veterinary Sciences, is 1.013 (2018). As a growing Journal, our goal in the mid-term is to position Austral Journal of Veterinary Sciences in the second quartile. During the last four years, our journal has experienced significant transformations with the only purpose to improve the quality of manuscripts that our readers deserve. Austral Journal of Veterinary Sciences has transitioned from being bilingual to English-only and has improved and enhanced the editorial processes by using an online platform. We expect to increase our audience, which requires work from the Editors to persuade researchers to submit articles of better quality. Therefore, many more challenges await our Editorial team in the short-term.

As an Open Access journal, reaching the community may come from different perspectives that are not being covered by the single parameter called Impact Factor. Thus, just as Journals have made progress and changes to increase their quality, readability, and visibility, including the Austral Journal of Veterinary Sciences, we expect no less from the Impact Factor. We shall wait and see how perfectible this metric can be.

Editorial Committee Austral Journal of Veterinary Sciences 